\title{
Velocity of Body Lean Evoked by Leg Muscle Vibration Potentiate the Effects of Vestibular Stimulation on Posture
}

\author{
O. DZURKOVÁ, F. HLAVAČKA \\ Institute of Normal and Pathological Physiology, Slovak Academy of Sciences, Bratislava, Slovak \\ Republic
}

Received September 17, 2007

Accepted October 1, 2007

\begin{abstract}
Summary
To investigate the vestibular and somatosensory interaction in human postural control, a galvanic vestibular stimulation of cosine bell shape resulting in a small forward or backward body lean was paired with three vibrations of both soleus muscles. The induced body lean was registered by the position of the center of foot pressure (CoP). During a quiet stance with eyes closed the vibration of both soleus muscles with frequency (of) $40 \mathrm{~Hz}, 60 \mathrm{~Hz}$ and $80 \mathrm{~Hz}$ resulted in the body lean backward with velocities related to the vibration frequencies. The vestibular galvanic stimulation with the head turned to the right caused forward or backward modification of CoP backward response to the soleus muscles vibration and peaked at 1.5-2 s following the onset of the vibration. The effect of the paired stimulation was larger than the summation of the vestibular stimulation during the quiet stance and a leg muscle vibration alone. The enhancement of the galvanic stimulation was related to the velocity of body lean induced by the leg muscle vibration. The galvanic vestibular stimulation during a faster body movement had larger effects than during a slow body lean or the quiet stance. The results suggest that velocity of a body postural movement or incoming proprioceptive signal from postural muscles potentiate the effects of simultaneous vestibular stimulations on posture.
\end{abstract}

\section{Key words}

Vestibular system • Postural control • Muscle vibration • Sensory interaction

The role of the human stance control is to maintain the vertical body's centre of gravity within the area of the feet support. Multisensory information, mainly from vestibular, somatosensory and visual afferents are integrated in a continual process of adjusting the body's centre of gravity in relation to an internal space reference frame (Maurer et al. 2006, Horak and MacPherson 1996).

Galvanic vestibular stimulation (GVS) can induce postural reactions in standing subjects that are useful in determining the influence of vestibular function on balance (Fitzpatrick and Day 2004). Application of electric current between the human mastoid processes during the stance with head turned to the right or left evokes the body lean mainly forward or backward towards the side of the anode (Lund and Broberg 1983). Proprioceptive input from postural muscles, particularly from leg muscles, is also important for balance control. In standing subjects, vibration of leg muscles results in a postural response known as the vibratory induced falling. These postural reactions can be characterized by body 
leaning to the direction of the vibrated muscles (Hayashi et al. 1981, Polónyová and Hlavačka 2001, Čapičíková et al. 2006).

Some studies have reported an influence of active body movements on the postural response to GVS, which was enhanced if the galvanic stimulus was applied during subject's lateral voluntary movements (SeveracCauquil and Day 1998). Similarly, postural response to a platform translation was altered in those trials in which GVS was applied during the translation (Inglis et al. 1995). This effect was greater than that produced by GVS alone in the absence of the platform translation. The largely summation effect of electrical vestibular stimulation and leg muscle vibration on the body lean has been already showed (Hlavačka et al. 1995). Asymmetric vestibular input provided by GVS during a symmetric vibration of hip abductors induced a lateral body sway (Popov et al. 1999).

In this study, we have examined the interaction between vestibular and proprioceptive signals in postural control. The aim was to find out how different velocity and magnitude of body lean induced by vibration of lower leg muscles would modify the magnitude of postural response to the galvanic vestibular stimulation.

Informed consent has been received from 10 healthy volunteers ( 6 males and 4 females) from 20 to 24 years of age (average $21.1 \pm 1.6$ years). Subjects stood on force platform with their eyes closed and the head turned towards the right shoulder. Their arms were held at body sides and feet were placed with heels together, opened at an angle of 30 degrees. They were instructed to keep their balance. To ensure that the initial stance position was the same prior to all trials, the anterior/posterior center of pressure (CoP) shifts were viewed on-line on the monitor.

Forward and backward postural responses to the galvanic vestibular stimulation were paired with three different vibrations of both soleus muscles and control condition was GVS without a vibration (together 12 conditions). Vibration with frequency of 40-60-80 Hz during the quiet stance with eyes closed resulted in backward body lean the velocity of which was related to the vibration frequencies. Vibratory stimulation (duration $5 \mathrm{~s}$, frequency $40 \mathrm{~Hz}, 60 \mathrm{~Hz}$ and $80 \mathrm{~Hz}$ and amplitude $1 \mathrm{~mm}$ ) was sequentially applied on the muscle belly of both soleus muscles. A small DC-motor with unbalanced load (5 g) was used as a vibrator. The vibrator was fastened to the muscles of the lower legs with a rubber strap. Bipolar, binaural galvanic stimulation of the

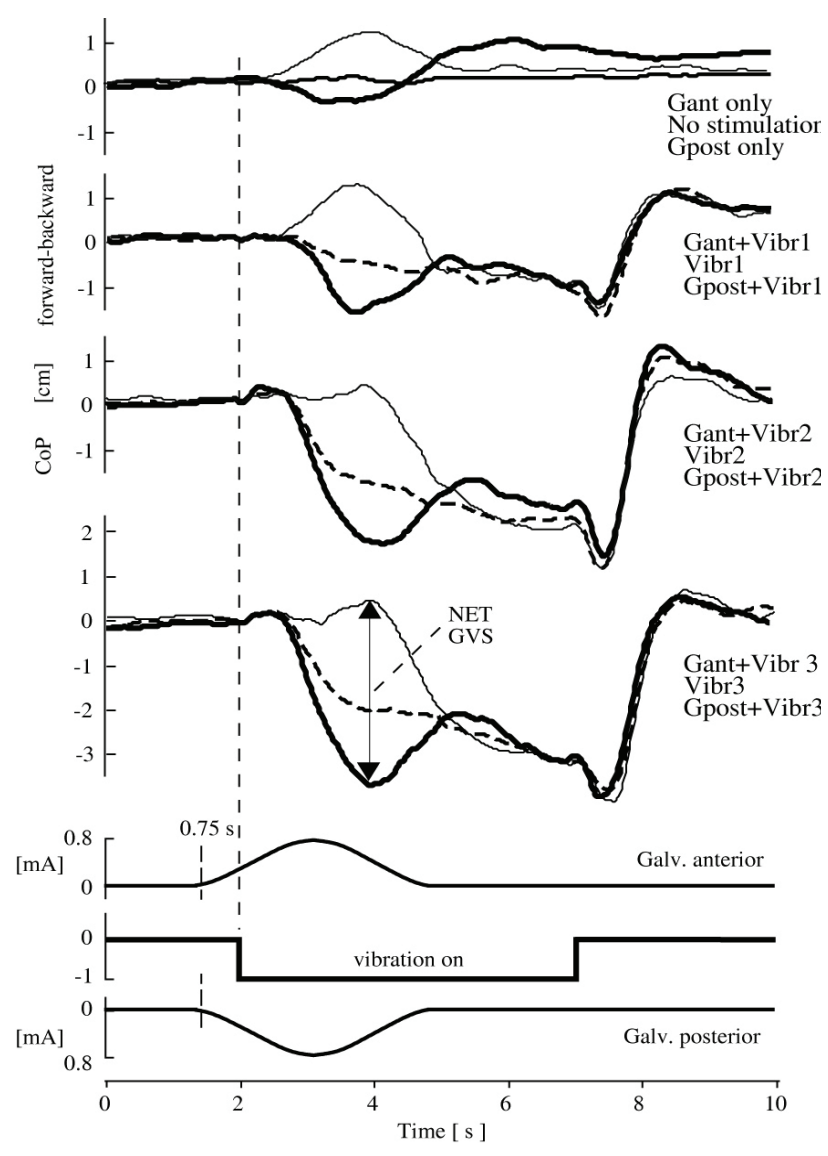

Fig. 1. The CoP responses to anterior (Gant - thin lines) and posterior (Gpost - thick lines) vestibular stimulation paired with three backward vibrations of soleus muscles (Vibr1 $40 \mathrm{~Hz}$, Vibr2 $60 \mathrm{~Hz}$, Vibr3 $80 \mathrm{~Hz}$ ) during the quiet stance. The net effect of GVS, which is characterized as a difference of CoP responses to GVS anterior and posterior, and with the frequency of vibration.

vestibular system was used with electrodes placed on the both mastoid processes. For vestibular "forward" stimulation, body lean forward, the positive electrode (anode) was placed on the left mastoid and a cathode on the right mastoid and head was turned to the right. For vestibular "backward" stimulation, polarities were reversed. Kidney-shaped, silver/silver chloride electrodes $\left(9 \mathrm{~cm}^{2}\right)$ were enveloped into gauze moistened with the physiological solution. A constant current stimulator produced the cosine bell-shaped galvanic current with peak value $0.8 \mathrm{~mA}$ and duration $3.3 \mathrm{~s}$. The cosine bell form of the galvanic stimulation was chosen because of its similarity to the actual, physiological change of vestibular information during the body inclination. Each vestibular stimulus was started $0.75 \mathrm{~s}$ prior to muscle vibration onset in order to evoke the peak sway response to the vestibular and vibration stimulations, simultaneously (Hlavačka et al. 1999).

The center of foot pressure $(\mathrm{CoP})$ of subjects in 
upright stance was measured by a force platform constructed on three pressure transducers and equipped with an automatic weight correction. The anteriorposterior direction was converted to a digital form at sampling frequency $50 \mathrm{~Hz}$. Data were stored and evaluated with a PC. The $\mathrm{CoP}$ records were analyzed in 10 -s intervals $-2 \mathrm{~s}$ before the vibration onset and $3 \mathrm{~s}$ after each 5-s vibration-stimulation interval. Every four postural responses during each experimental condition were averaged for each subject. Each subject's postural responses were adjusted so that the mean value of CoP positions for $2 \mathrm{~s}$ before the stimulation was considered as zero. The final postural response evoked by either galvanic or vibratory stimulation or their combination was averaged for all 10 subjects (10 records for a/the sagital component of body tilt $\mathrm{x} 4$ trials). The net postural effect of the galvanic vestibular stimulation was evaluated as a difference of $\mathrm{CoP}$ responses to anterior (Gant) and posterior (Gpost) vestibular stimulation during the quiet stance. The paired Student's $t$-test was used to analyze differences between conditions with and without the vibration of muscles.

Figure 1 shows that vibration of soleus muscles induced changes in the position of the CoP (dashed lines), which started with a faster increase in magnitude and reached a plateau (position). The 5-s vibration of both soleus muscles resulted in the body lean backward. The averaged magnitudes of the $\mathrm{CoP}$ position shift measured at $2 \mathrm{~s}$ following the onset of vibration were $1.08 \pm 0.26 \mathrm{~cm}$ backward during the $40 \mathrm{~Hz}$ vibration, $1.61 \pm 0.29 \mathrm{~cm}$ backward during the $60 \mathrm{~Hz}$ and $2.43 \pm 0.33 \mathrm{~cm}$ backward during the $80 \mathrm{~Hz}$ soleus vibration. Vestibular stimulation produced anterior-posterior shifts in the position of the CoP with the direction depending on the polarity of the galvanic current and then back to the initial position (Fig. 1 , thick and thin lines). The averaged maximal changes of CoP position induced by galvanic stimulation during the stance without a muscle vibration were $1.41 \pm 0.20 \mathrm{~cm}$ forward and $0.73 \pm 0.13 \mathrm{~cm}$ backward (Fig. 1). The magnitudes of the CoP position shifts produced by vestibular and proprioceptive stimulation were similar.

Comparison of CoP curves with only proprioceptive vibration to the paired vestibular and proprioceptive stimulation has shown that the CoP magnitude was increased during the combined stimulation. Summation of effects of the vestibular stimulation alone and proprioceptive stimulation alone resulted in the increased magnitude of the CoP curves, particularly in the cases where the frequency of muscle

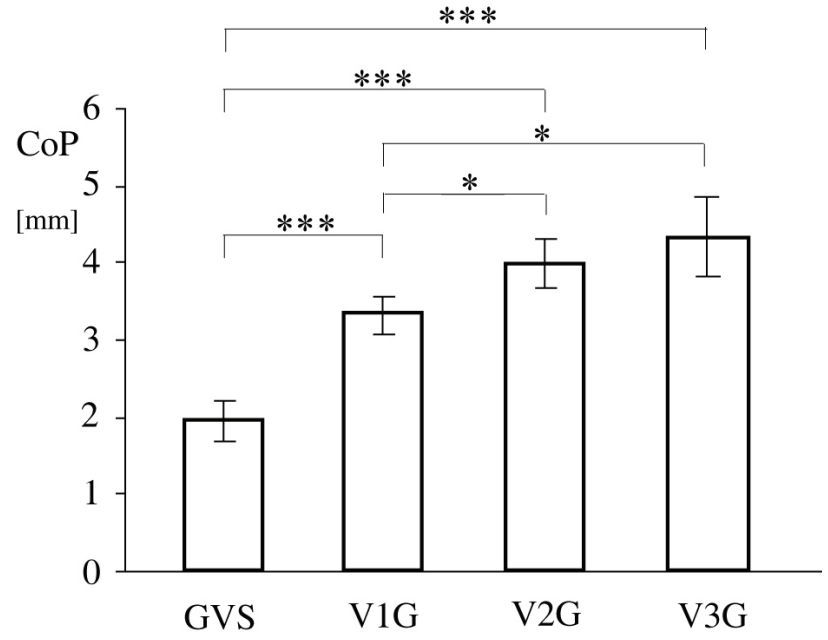

Fig. 2. Net effects of the galvanic vestibular stimulation without vibration (GVS) and paired with three backward vibrations of soleus muscles (V1G-40 Hz, V2G-60 Hz, V3G-80 Hz) estimated as a difference of CoP responses to anterior (Gant) and posterior (Gpost) vestibular stimulation during quiet stance. The data are mean \pm S.E.M. $(n=10)$.

vibration was higher (Fig. 1). The larger and significant effect of galvanic vestibular stimulation on the response to the lower leg muscle vibration was evident using the parameter of (the) „GVS net effect“" (Fig. 2).

The effect of galvanic stimulation during a vibration of soleus muscles was not the simple addition of a vestibulospinal response during the quiet stance and a postural response to the proprioceptive stimulation of lower leg muscles. When the single response to a vibration of both soleus muscles was subtracted from the combined vestibular stimulation and muscles vibration, the effect of the vestibular stimulation (Fig. 2 - V1G, $\mathrm{V} 2 \mathrm{G}, \mathrm{V} 3 \mathrm{G})$ was larger than the effect of vestibular stimulation during the quiet stance (Fig. 2 - GVS). The influence of the vestibular stimulation was larger the faster the body was leaned by muscles vibration, suggesting that the vestibular information may be more critical when controlling fast body motions than the slow ones or a quiet stance (Hlavačka and Horak 2006).

The postural control system has two main functional goals that may be controlled independently: orientation and equilibrium (Horak and MacPherson 1996). Maintaining the orientation requires the interpretation of sensory information to achieve the relative alignment of body with a reference to the environment, which includes surface contacts, gravitoinertial forces and also proprioceptive information (Kavounoudias et al. 1999). Maintaining the equilibrium requires keeping the center of body mass balanced over 
the base of a support. The postural responses, which restore the equilibrium in the face of external or internal perturbations, must operate upon some particular orientation, i.e. an initial body position and an internally represented orientation goal. In our study, equilibrium responses induced by somatosensory inputs (muscles vibration) were superimposed upon an internal reference for the vertical that was altered by the galvanic vestibular stimulation (Hlavačka et al. 1995, Popov et al. 1999).

Our study has shown that the effect of the paired stimulation has been larger than the summation of the vestibular stimulation during the quiet stance and leg muscle vibration alone. The enhancement of the galvanic stimulation was related to velocity and magnitude of the body lean induced by the leg muscle vibration. Galvanic vestibular stimulation during an increased proprioceptive signal from lower leg muscles had larger effects than during the quiet stance. Our results suggest that body postural movement or incoming proprioceptive signal from postural muscles potentiate the effects of simultaneously vestibular stimulation on posture.

\section{Acknowledgements}

This work was supported by Slovak grant agency VEGA No.2/4070/27.

\section{References}

ČAPIČÍKOVÁ N, ROCCHI L, HLAVAČKA F, CHIARI L, CAPPELLO A: Human postural response to lower leg muscle vibration of different duration. Physiol Res 55 (Suppl 1): S129-S134, 2006.

FITZPATRICK R, DAY BL: Probing the human vestibular system with galvanic stimulation. $J$ Appl Physiol 96: 23012316, 2004.

HAYASHI R, MIYAKE A, JIJIWA H, WATANABE S: Postural readjustment to body sway induced by vibration in man. Exp Brain Res 43: 217-225, 1981.

HLAVAČKA F, KRIŽKOVÁ M, HORAK FB: Modification of human postural response to leg muscle vibration by electrical vestibular stimulation. Neurosci Lett 189: 9-12, 1995.

HLAVAČKA F, SHUPERT CL, HORAK FB: The timing of galvanic vestibular stimulation affects responses to platform translation. Brain Res 821: 8-16, 1999.

HLAVAČKA F, HORAK FB: Somatosensory influence on postural response to galvanic vestibular stimulation. Physiol Res 55 (Suppl 1): S121-S127, 2006.

HORAK FB, MACPHERSON JM: Postural orientation and equilibrium. Exercise: regulation and integration of multiple systems. In: SHEPHERD J, ROWELL L (eds.): Handbook of Physiology, Oxford University Press, New York, 1996, pp 255-292.

INGLIS JT, SHUPERT CL, HLAVAČKA F, HORAK FB: The effect of galvanic vestibular stimulation on human postural responses during support surface translations. J Neurophysiol 73: 896-901, 1995.

KAVOUNOUDIAS A, GILHODES JC, ROLL R, ROLL JP: From balance regulation to body orientation: two goals for muscle proprioceptive information processing? Exp Brain Res 124: 80-88, 1999.

LUND S, BROBERG C: Effects of different head positions on postural sway in man induced by a reproducible vestibular error signal. Acta Physiol Scand 117: 307-309, 1983.

MAURER C, MERGNER T, PETERKA RJ: Multisensory control of human upright stance. Exp Brain Res 171: 231$250,2006$.

POLÓNYOVÁ A, HLAVAČKA F: Human postural responses to different frequency vibrations of lower leg muscles. Physiol Res 50: 405-410, 2001.

POPOV KE, KOZHINA B, SMETANIN N, SHLIKOV VY: Postural response to combined vestibular and hip proprioceptive stimulation in man. Eur J Neurosci 11: 3307-3311, 1999.

SEVERAC-CAUQUIL A, DAY BL: Galvanic vestibular stimulation modulates voluntary movement of the human upper body. J Physiol Lond 513: 611-619, 1998.

\section{Corresponding author}

F. Hlavačka, Institute of Normal and Pathological Physiology, Slovak Academy of Science, Sienkiewiczova 1, 81371 Bratislava, Slovak Republic. Fax: +421 252968 516. E-mail: frantisek.hlavacka@savba.sk 